\title{
A comparative study on the effect of four disinfectant solutions on wettability of three types of elastomeric impression materials with two different immersion time: An in-vitro study
}

\author{
Vijaya Kumar R ${ }^{1}$, M Viswambaran ${ }^{2}$, Parag Dua ${ }^{3 *}$, Poonam Prakash ${ }^{4}$ \\ ${ }^{1}$ Reader, ${ }^{2}$ Professor, ${ }^{3,4}$ Associate Professor, ${ }^{1.3 .4}$ Armed Forces Medical College, Pune, Maharashtra, ${ }^{2}$ Army Dental Centre \\ (R\&R), New Delhi, India
}

\section{*Corresponding Author: Parag Dua}

Email: duaparag@gmail.com

\begin{abstract}
Introduction: Infection control is an important concept in the present day practice of dentistry. Elastomeric dental impressions too should be disinfected as per laid down protocols. Recently, vinylsiloxaether type of elastomeric impression material have been introduced which is supposed to have improved hydrophilic properties among others. This invitro study was taken up to compare wettability of vinylsiloxaether impression material in comparison to two other popular traditional elastomeric impression materials i.e polyether and addition silicone.

Materials and Methods: An in-vitro study was undertaken to evaluate effect of four recommended commercially available disinfectant solutions on the wettability of three types of elastomeric impression materials at 10 and 30 minutes time intervals. The impression materials evaluated were vinylsiloxaether, addition silicone and polyether. The disinfectants used were $2 \%$ glutaraldehyde, $0.5 \%$ sodium hypochlorite, $0.05 \%$ iodophor and $0.25 \%$ benzalkonium chloride. 100 samples of each impression material was made and subjected to 10 different immersion protocols including distill water as control. The surface wettability (contact angle) of all samples was measured using the contact angle analyzer (Digidrop, Contact angle meter, GBX products, France). The data obtained was statistically analyzed. 'Two-sample $t$-tests', 'two- way- ANOVA' and 'tukey simultaneous test' was applied.

Results: The mean contact angle values of addition silicone samples were highest among elastomers followed by polyether and vinylsiloxaether samples. The mean value of contact angles for 30 minutes immersion time interval was highly significant compared to the corresponding values of 10 minutes immersion time interval $(\mathrm{P}<0.0001)$. In each case, the mean contact angles were higher for 30 minutes immersion time interval.

Conclusion: Among the elastomeric impression materials evaluated, wettability wise vinylsiloxaether proved to be the best impression material followed by polyether and addition silicone. Disinfection with $2 \%$ glutaraldehyde had shown lowest contact angle values followed by $0.5 \%$ sodium hypochlorite solution, $0.25 \%$ benzalkonium chloride and $0.05 \%$ iodophor respectively.
\end{abstract}

Keywords: Disinfection, Vinylsiloxaether, Wettability, Contact angle, Elastomers.

\section{Introduction}

Dental impression making is a routine dental procedure in all dental surgeries and more often contamination of dental impressions with varying amount of blood and saliva does occur. Therefore these impressions have the potential to transmit serious diseases to all dental personnel who routinely handle them. Furthermore it has been shown that contaminated impressions can cross infect gypsum casts that have been poured against them. It is therefore imperative that standard protocols for disinfecting dental impressions are followed for all patients. ${ }^{1-3}$

Infection control is an important concept in the present day practice of dentistry. Disinfection is preferred for impression materials over sterilization by many authors. Preference is given to disinfection procedures based on the fact that these materials need to be sterile since they are not implanted into a sterile body cavity, but rather are inserted in and withdrawn from the oral cavity. ${ }^{4,5}$

Most dental practioners currently use primarily polyether and addition elastomeric impression 
materials in one-step impression technique applications. Advances in elastomeric impression material chemistries have lead to introduction of new generation impression materials like combination of a polyvinyl and a polyether impression material called vinylsiloxaether. As per the manufacturer it combines some of the most desired properties of both materials into one material. Vinylsiloxaether is supposed to be extremely hydrophilic and has excellent flowability.

Hydrophilicity is one of the most desirable and essential properties of an impression material. Wettability is defined as relative affinity of a liquid for the surface of a solid. The wettabilty of a surface is usually determined by measuring the magnitude of contact angle formed between a drop of liquid and the surface in question. Small contact angles indicate good Wettability. A problem with use of use of elastomeric impression materials is their low surface energy, which makes it difficult to wet the impression materials with gypsum slurry. A literature review failed to reveal studies which assessed the effect of disinfectants on wettabilty of vinylsiloxaether impression material in comparison to other types of elastomeric impression materials. ${ }^{11-17}$

The present study was therefore undertaken to evaluate the effect of four recommended commercially available disinfectant solutions on the wettability of three different types of impression materials with two different recommended time intervals (10 minutes and 30 minutes).

\section{Materials and Methods}

In this study three impression materials were evaluated i.e Vinylsiloxaether (Identium $^{\circledR}$ Light, Kettenbach $\mathrm{GmbH} \& \mathrm{Co} . \mathrm{KG}$ ), Addition silicone $\left(\right.$ Betasil $^{\circledR}$ vario light, Müller-Omicron, Germany) and Polyether (Impregnum TM soft, 3M ESPE ${ }^{\circledR}$, USA, Dental products, St Paul, MN 55144-1000). All materials used in this study were low-viscosity materials in an effort to achieve a homogeneous void-free mix. For the fabrication of the samples a custom fabricated mould was used of $30 \mathrm{~mm}$ diameter and $3 \mathrm{~mm}$ width (Fig. 1).

The impression materials were dispensed and mixed as per manufacturer's specifications. The custom made metal mould was cleaned with $99 \%$ ethanol and placed on a glass slab over which a polyethylene strip of same size had been placed. The appropriately mixed impression materials were loaded in the mould. Care was taken during filling of the mould to avoid incorporation of air bubbles. Another polyethylene strip \& clean glass slab was placed immediately on the over filled mold. A clamp was used to apply uniform pressure on top of the overfilled mould. Impression samples were allowed to set for time duration as prescribed by the manufacturer before separation from the mould. All the samples were inspected and those not upto the specification were discarded and remade. The impression material samples were handled with forceps and the operator wore nitrile gloves throughout the procedures to avoid any contamination. A total of 100 disc shaped flat surface samples were prepared of each impression material.

Four ADA recommended disinfectants were used in this study. The disinfectants used were $2 \%$ glutaraldehyde, $0.5 \%$ sodium hypochlorite, $0.05 \%$ iodophor and $0.25 \%$ benzalkonium chloride. The distilled water was prepared using distil water plant and was used as the control.

The impression materials were grouped into three groups and disinfectants were categorized into four categories with control distilled water forming the fifth category. 100 samples of each group were prepared using the custom fabricated mould. After preparation of the samples for different impression materials, they were disinfected in requisite concentration of disinfectants, following ten different protocols (Table 1). 10 samples of respective impression material was subjected to immersion disinfection in all four selected disinfectant and distilled water for two time durations i.e 10 minutes and 30 minutes respectively. After completion of disinfection protocol of each impression material, they were evaluated for their wettability by measuring the contact angle of each sample using a contact angle analyzer (Fig. 2).

For measuring contact angle a saturated solution of calcium sulphate dihydrate was used. ${ }^{7,8,11}$ Before and after disinfection, the samples are rinsed for 10 seconds. To determine the contact angle equal size drops of saturated solution of calcium sulphate dihydrate i.e $0.05 \mathrm{ml}$ were dispensed over the surface 
of the sample using calibrated microburette (Fig. 3). The image of the drop of the solution contacting the sample (interface) was captured automatically by the camera within 30 seconds. Two contact angle readings were taken for each drop at either ends of the image (right and left) of the drop by the computer software (Fig. 4). Average of both the readings was calculated to get the final reading of contact angle for each sample by the computer software.

The data obtained was statistically analyzed and the mean and standard deviation were calculated for each group. 'Two-sample $t$-tests', 'two-way-ANOVA' and 'tukey simultaneous test' was applied. For statistical analysis, the statistical software 'MINITAB13' was used.

\section{Results}

After analyzing the results, it was seen that mean contact angle values of addition silicone samples were highest among impression materials followed by polyether and vinylsiloxaether samples.

The comparison between mean contact angles for the two immersion time intervals, for each impression material subjected to disinfectant glutaraldehyde and sodium hypochlorite, the mean value of contact angles for 30 minutes immersion time interval was highly significant compared to the corresponding values of 10 minutes immersion time interval $(\mathrm{P}<0.0001)$. In each case, the mean contact angles were higher for 30 minutes immersion time interval (Table 2 and 3).

Table 4 presents the comparison between mean contact angles for the two immersion time intervals, for each impression material subjected to disinfectant iodophor. Here for vinylsiloxaether (VS group) the difference between mean contact angles for 10 minutes and 30 minutes immersion time interval were not statistically significant $(\mathrm{T}$-value $=0.55$ and $\mathrm{P}$-value $=$ 0.5890 ). For impression material addition silicone (AS group) the differences between immersion time intervals were highly significant $(\mathrm{T}$-value $=6.18$ and P-value $=0.0001)$; however, the trend was different here. The mean value for 10 minute period (103.26) was statistically higher than the 30 minute period (99.42). With impression material polyether (PE group) the immersion time intervals differ at only $5 \%$ level $(\mathrm{T}$ - value $=2.30 \& \mathrm{P}$-value $=0.0380)$.

Table 5 depicts the comparison between mean contact angles for the two immersion time intervals, for each impression material subjected to disinfectant benzalkonium chloride. The table shows means, standard deviations, T-values and corresponding Pvalues of the respective impression materials. The Tvalue and P-value for different impression materials were as follow: VS group (T-value $=0.34 \& \mathrm{P}$-value $=0.7350)$, AS group $(\mathrm{T}$ - value $=2.03 \& \mathrm{P}$-value $=$ $0.0580)$ and $\mathrm{PE}$ group $(\mathrm{T}$ - value $=1.15 \& \mathrm{P}$-value $=$ 0.2640). Among the elastomers the differences in $\mathrm{T}$ value and $P$-value were statistically not significant.

At 10 minutes immersion time interval, all pair wise comparisons among all disinfectants including control (distilled water) were statistically significant ( $\mathrm{P}$ $=0.00001)$. It was observed that overall the mean contact angle for control (distilled water) was lowest and among the disinfectants lowest mean contact angle were for sodium hypochlorite followed by glutaraldehyde and benzalkonium chloride. The mean contact angle value among disinfectants was highest for disinfectant iodophor.

At 30 minutes immersion time interval, all the pair wise comparisons were again statistically significant $(\mathrm{P}=0.00001)$ except in the case of the comparison between disinfectants iodophor and benzalkonium chloride where the level was little lower ( $\mathrm{P}=0.00040)$. As in the case of immersion time interval of 10 minutes, the control (distilled water) gives statistically significant lowest contact angle when compared to all disinfectants. Among the disinfectants the lowest mean contact angles were for glutaraldehyde followed by sodium hypochlorite and benzalkonium chloride. The mean contact angle value among disinfectants was highest for disinfectant iodophor. 
Table 1: Disinfection protocol for group - VS (vinylsiloxaether impression material)

\begin{tabular}{|c|c|c|c|}
\hline Method & Subgroups & Disinfection protocol & No. of Samples \\
\hline I & VS,GD, 10 & $\begin{array}{l}\text { vinylsiloxaether specimens } \\
\text { gluteraldehyde for } 10 \text { minutes }\end{array}$ & 10 \\
\hline II & $\mathrm{VS}, \mathrm{GD}, 30$ & $\begin{array}{llll}\begin{array}{l}\text { vinylsiloxaether specimens } \\
\text { gluteraldehyde for } 30 \text { minutes }\end{array} & & \text { immersed } & 2 \% \\
\end{array}$ & 10 \\
\hline III & VS,SH,10 & $\begin{array}{l}\text { vinylsiloxaether specimens immersed in } 0.5 \% \\
\text { sodium hypochlorite for } 10 \text { minutes }\end{array}$ & 10 \\
\hline IV & VS,SH,30 & $\begin{array}{l}\text { vinylsiloxaether specimens immersed in } 0.5 \% \\
\text { sodium hypochlorite for } 30 \text { minutes }\end{array}$ & 10 \\
\hline V & VS,IO,10 & $\begin{array}{l}\text { vinylsiloxaether specimens immersed in } 0.05 \% \\
\text { iodophor for } 10 \text { minutes }\end{array}$ & 10 \\
\hline VI & VS,IO,30 & $\begin{array}{l}\text { vinylsiloxaether specimens immersed in } 0.05 \% \\
\text { iodophor for } 30 \text { minutes }\end{array}$ & 10 \\
\hline VII & VS,BC, 10 & $\begin{array}{l}\text { vinylsiloxaether specimens immersed in } 0.25 \% \\
\text { benzalkonium chloride for } 10 \text { minutes }\end{array}$ & 10 \\
\hline VIII & VS,BC,30 & $\begin{array}{l}\text { vinylsiloxaether specimens immersed in } 0.25 \% \\
\text { benzalkonium chloride for } 30 \text { minutes }\end{array}$ & 10 \\
\hline IX & VS,DW,10 & $\begin{array}{l}\text { vinylsiloxaether specimens left untreated without } \\
\text { disinfection immersed in distilled water for } 10 \\
\text { minutes }\end{array}$ & 10 \\
\hline $\mathrm{X}$ & VS,DW,30 & $\begin{array}{l}\text { vinylsiloxaether specimens left untreated without } \\
\text { disinfection immersed in distilled water for } 30 \\
\text { minutes }\end{array}$ & 10 \\
\hline
\end{tabular}

NOTE: Protocols for other two materials i.e for PE (polyether) and AS (Addition silicone) are similar

Table 2: Mean / std dev: contact angle; $(\mathrm{N}=10$ for each cell)

\begin{tabular}{|c|l|c|c|c|c|c|}
\hline Immersion & Impression & \multicolumn{5}{|c|}{ Disinfectants } \\
\hline Time & Materials & $\mathbf{2 \%}$ GD & $\mathbf{0 . 5 \%}$ SH & $\mathbf{0 . 0 5 \%}$ IO & $\mathbf{0 . 2 5 \%}$ BC & Control ( DW) \\
\hline \multirow{3}{*}{ 10 Minutes } & PE & $72.55 / 1.3$ & $74.98 / 1.98$ & $87.82 / 1.46$ & $84.87 / 1.16$ & $62.50 / 0.85$ \\
\cline { 2 - 7 } & $\mathbf{A ~ S ~}$ & $97.20 / 0.79$ & $93.26 / 0.53$ & $103.26 / 1.43$ & $101.67 / 0.94$ & $74.74 / 0.57$ \\
\cline { 2 - 7 } & $\mathbf{V S}$ & $37.70 / 0.87$ & $44.21 / 1.12$ & $57.22 / 0.82$ & $53.80 / 1.67$ & $33.57 / 1.26$ \\
\hline \multirow{3}{3}{ Minutes } & $\mathbf{P E}$ & $76.55 / 1.42$ & $86.09 / 1.33$ & $88.30 / 2.33$ & $85.20 / 2.81$ & $64.04 / 0.57$ \\
\cline { 2 - 7 } & $\mathbf{A ~ S ~}$ & $102.56 / 0.71$ & $101.81 / 0.74$ & $99.42 / 1.35$ & $102.35 / 0.49$ & $77.06 / 0.99$ \\
\cline { 2 - 7 } & $\mathbf{V S}$ & $41.50 / 2.67$ & $50.90 / 1.24$ & $58.38 / 1.37$ & $54.66 / 1.67$ & $36.34 / 0.13$ \\
\hline
\end{tabular}

Table 3: Overall mean and std dev: each factor (level wise)

\begin{tabular}{|l|l|c|r|r|}
\hline \multicolumn{1}{|c|}{ Factors } & \multicolumn{1}{|c|}{ Levels } & N & Mean & \multicolumn{1}{c|}{ S. D. } \\
\hline \multirow{3}{*}{ Immersion Time } & 10 Minutes & 200 & 73.56 & 19.82 \\
\cline { 2 - 5 } & 30 Minutes & 200 & 76.95 & 19.65 \\
\hline Impression Material & Polyether (PE) & 100 & 79.25 & 9.36 \\
\cline { 2 - 5 } & Addition Silicon (AS) & 100 & 95.33 & 10.23 \\
\cline { 2 - 5 } & vinylsiloxaether (VS) & 100 & 46.83 & 8.64 \\
\hline
\end{tabular}




\begin{tabular}{|l|l|r|r|r|}
\hline Disinfectant & 2\% Glutaraldehyde (GD) & 80 & 75.05 & 22.62 \\
\cline { 2 - 5 } & 0.5\% Sodium Hydrochloride (SH) & 80 & 76.55 & 18.72 \\
\cline { 2 - 5 } & 0.05\% Iodophor (IO) & 80 & 82.95 & 16.05 \\
\cline { 2 - 5 } & O,25\% Benzal. Chloride (BC) & 80 & 80.97 & 17.37 \\
\cline { 2 - 5 } & Control (DW: Distilled Water) & 80 & 60.76 & 15.75 \\
\hline
\end{tabular}

Table 4: GLM: three factor analysis

\begin{tabular}{|l|lcl|}
\hline Factor & type & levels & values \\
\hline Time (minutes) & Fixed & 2 & 10,30 \\
Material & Fixed & 3 & PE, AS, VS \\
Disinfectant & Fixed & 5 & BC, DW, GD, IO, SH \\
\hline
\end{tabular}

\section{ANOVA}

\begin{tabular}{|l|c|c|c|c|c|}
\hline \multicolumn{1}{|c|}{ Source } & DF & SS & MS & F & P \\
\hline Time & 1 & 1147.9 & 1147.9 & 6.95 & $0.0486^{*}$ \\
\hline Material & 3 & 124867.0 & 41622.3 & 113.74 & $0^{\#}$ \\
\hline Disinfectant & 4 & 24280.8 & 6070.2 & 11.98 & $0.0097^{* *}$ \\
\hline Time* Material & 3 & 43.1 & 14.4 & 0.49 & $0.1322^{\mathrm{NS}}$ \\
\hline Time* Disinfectant & 4 & 619.9 & 155.0 & 5.39 & $0.0130^{*}$ \\
\hline Material* Disinfectant & 12 & 4221.8 & 351.8 & 12.13 & $0.0001^{* * * *}$ \\
\hline Time* Material *Disinfectant & 12 & 347.6 & 29.0 & 16.12 & $0.0001^{* * * *}$ \\
\hline Error & 360 & 646.9 & 1.8 & & \\
\hline Total & 399 & 156175.1 & & & \\
\hline
\end{tabular}

NOTE: NS: Not Significant; *: Significant at 5\%; **: Significant at 1\%; ***: Significant at 0.1\%; ****: Significant at $0.01 \%$; \#: Significant at $0 \%$

Table 5: Student's t test between time-10 minutes $x$ material VS $x$ disinfectant DW and time-10 minutes $x$ material VS $\mathrm{x}$ disinfectant $2 \%$ GD

\begin{tabular}{|l|c|c|c|c|}
\hline \multicolumn{1}{|c|}{ Combinations } & Mean \pm SD & Diff $($ Means) & t-Value & p-Value \\
\hline 10Min X VS X DW & $33.57^{0} \pm 1.26^{0}$ & 4.13 & 26.85 & 0.00001 \\
\hline 10Min X VS X 2\% GD & $37.70 \pm 0.87$ & & & \\
\hline
\end{tabular}

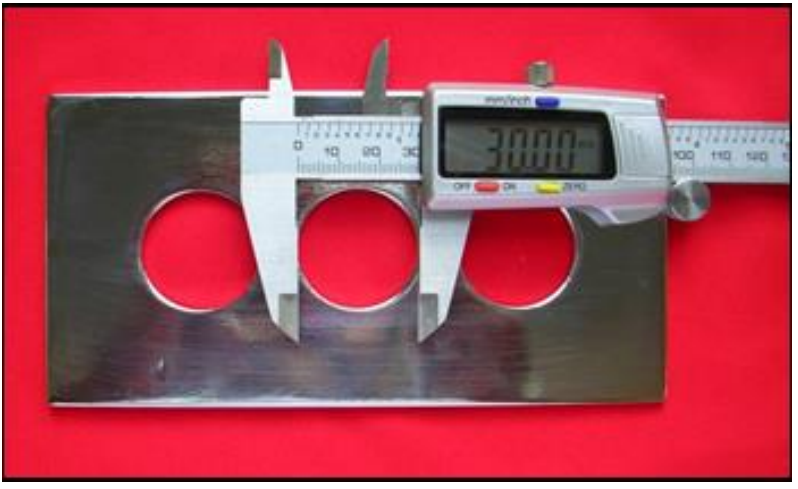

Fig. 1: Custom fabricated mould

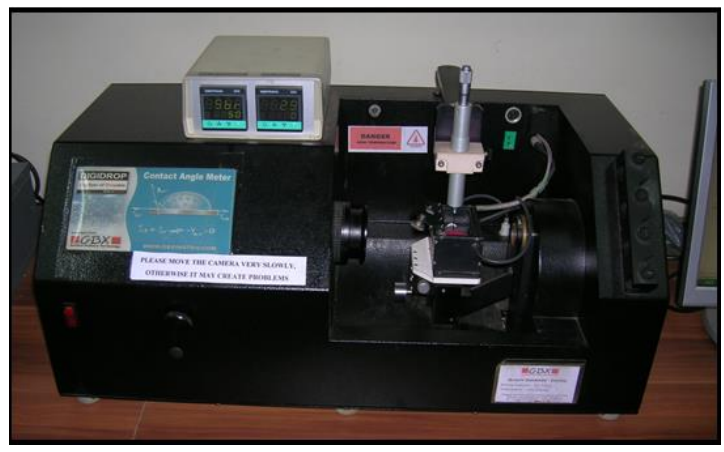

Fig. 2: Contact angle analyzer (Digidrop, Contact angle meter, GBX products, France) 


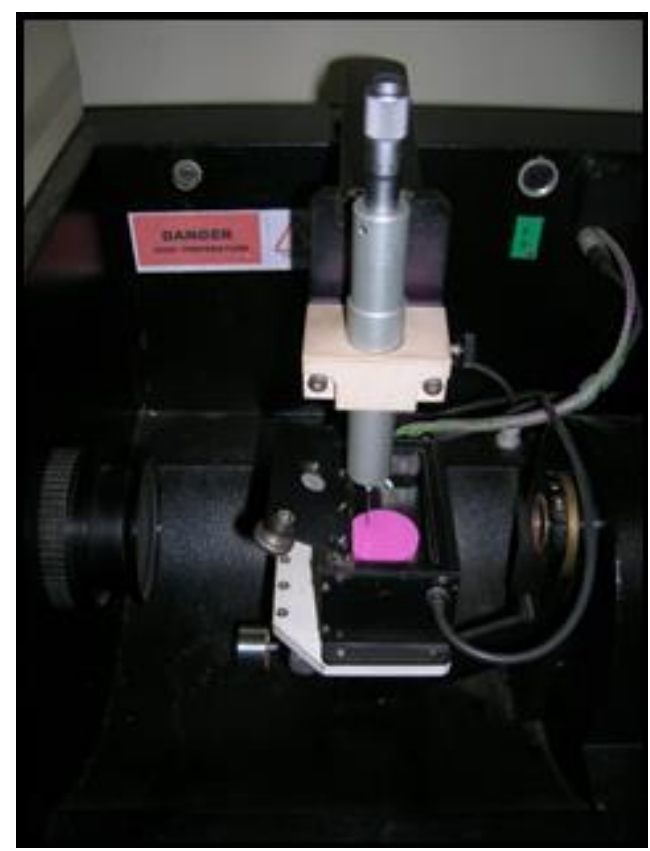

Fig. 3: Saturated solution of calcium sulphate dihydrate dispensed over the surface of the sample using calibrated microburette

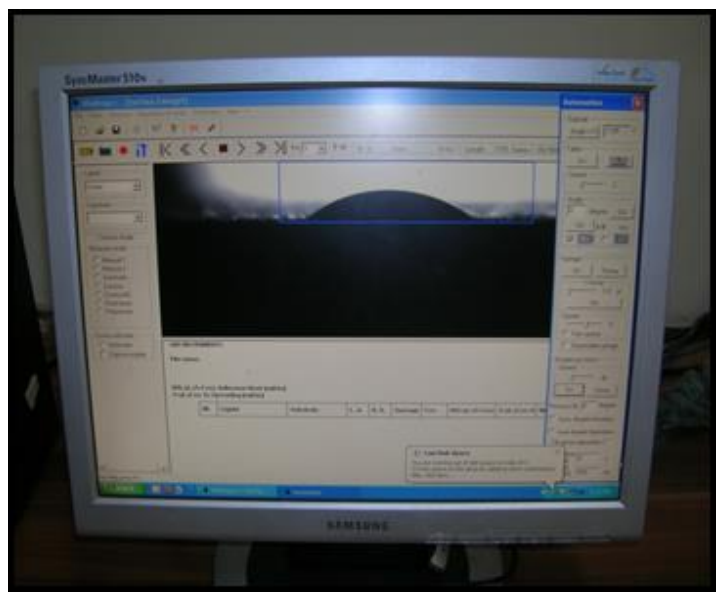

Fig. 4: Contact angle being analyzed and measured

\section{Discussion}

Dental impressions have the potential to transmit serious diseases to all dental personnel who routinely handle them. The routine procedure of rinsing impressions under tap water immediately after removal from the mouth eliminates gross contamination along with saliva and blood but not all microorganisms are removed and they can be a source of infection. There appears to be a great deal of conflict surrounding the impression disinfection techniques being used by dental offices and laboratories. Although various Governmental and private organizations like ADA (American Dental Association); OSHA (Occupational and Safety Hazards Organization); CDC (Centre for Disease Control, Government of United States, Department of labor) and dental literature provide guidance about how specific impression materials should be disinfected to balance the goals of safety and accuracy, they cannot offer definitive answers to the problems at hand because there is no faultless universal disinfectant. ${ }^{1-6}$

Disinfection eliminates virtually all recognized pathogenic microorganisms but not necessarily all microbial forms, on inanimate objects. Different methods for disinfection of impression materials have been suggested that includes argon radiofrequency glow discharge, ethylene oxide, autoclave, microwave, ultraviolet radiation and chemical disinfectant solutions ${ }^{\mathbf{1 - 5}}$. Among the different methods mentioned above, chemical disinfection is more commonly employed. Various agents used for chemical disinfection include alcohols, chlorine and chlorine compounds, formaldehyde, glutaraldehyde, orthophthalaldehyde, hydrogen peroxide, iodophors, peracetic acid, phenolics, and quaternary ammonium compounds. Disinfectants are available as spray and immersion solutions in different concentrations. ${ }^{1-6}$

Immersion disinfection is the most reliable method as it guarantees that all surfaces of impression and impression tray will come into contact with disinfectant solution. In 1991 ADA released new guidelines that recommended immersion disinfection for all the impression materials provided recommended time of disinfection is used. ${ }^{1-6}$ The contact time for the various products used as disinfectants showed variations from 3 to 30 minutes. ${ }^{1-}$

${ }^{6}$ Published reports in the dental literature often are varied regarding the time of immersion and concentrations. Therefore it is better to follow manufacturer's instructions before selecting a specific disinfectant. $^{1,2}$ Traditionally the iodophors, chlorine compounds, glutaraldehydes, phenols, benzalkonium chloride have required exposure time ranging from 10 to 30 minutes. The manufacturer's recommended exposure time for given disinfectant should be interpreted as the minimum exposure time. However 
the time may be exceeded if necessary but may not be reduced. The recommended concentrations varied depending on the manufacturer and type of disinfectants. The preferred concentrations of commonly used disinfectants are as follows - Sodium hypochlorite: 0.5 to $1 \%$, glutaraldehyde: 2 to $3.2 \%$, povidine-iodine: $\quad 0.1 \%$, formaldehyde: $4 \%$, chlorhexidine: $0.5 \%$, ethanol: $50 \%$, formalin: $10 \%$, benzalkonium chloride: $0.25 \% .^{1-6}$

Although elastomeric impression materials offer number of advantages for routine clinical procedures, one of the drawbacks of these materials is poor wettability. Inspite of repeated claims by the manufacturer that their material is superior and hydrophilic, we routinely encounter impression surface defects due to poor wettability. Vinylsiloxanether was specially developed for the onestep impression technique and combines the benefits of two established impression materials, polyether and addition-silicone materials, creating an entirely new material. The manufacturer claims they provide excellent flowability, exhibit improved elastic properties with dimensionally accurate recovery and more importantly have superior hydrophilicty. Wettability is defined as the ability of a liquid to spread over the surface of the solid. Contact angle or wetting angle is the angle formed at the interface between the droplet and the horizontal surface. A liquid is considered to be wetting a surface when contact angle is less than 90 degrees and is considered non-wetting when contact angle is more than 90 degrees. Thus an impression material is considered hydrophilic if the contact angle is less than 90 degrees. $^{3,4}$ There is an ongoing effort by dental manufacturers to create impression materials with improved wetting properties. Disinfection solutions may alter the surface characteristics of these newer materials. ${ }^{15-20}$

When the impression materials were evaluated for mean contact angles after immersion in various disinfectants for 10 and 30 minutes immersion time intervals, it was seen that the mean contact angle values for Identium ${ }^{\circledR}$ Light, Kettenbach $\mathrm{GmbH} \&$ Co. KG (vinylsiloxaether) showed highest wettability compared to Impregnum ${ }^{\mathrm{TM}}$ soft (polyether) and Betasil $^{\circledR}$ vario light (addition silicone) after being subjected to $2 \%$ glutaraldehyde, $0.5 \%$ sodium hypochlorite, $0.05 \%$ iodophor and $0.25 \%$ benzalkonium chloride including control (distilled water). It was seen that overall for all disinfection protocols the subgroups of vinylsiloxaether showed the better wettability results followed by polyether and addition silicone. All the elastomeric impression materials evaluated in this study were found to be hydrophilic since their contact angle values were less than 90 degrees when subjected to control (distilled water). The mean contact angle values for vinylsiloxaether remained below 90 degrees after subjecting to four different disinfectants. The mean contact angle for Impregnum ${ }^{\mathrm{TM}}$ soft (polyether) also remained below 90 degrees after being subjected to all disinfectants. However the contact angle values between vinylsiloxaether and polyether silicone differed substantially, implying vinylsiloxaether is more hydrophilic than polyether. Betasil ${ }^{\circledR}$ vario light (addition silicone) also can be considered as hydrophilic as the contact angle value were close to 90 degrees and remained below 100 degrees after being subjected to all the disinfectant protocols.

When the mean contact angle values of all the impression materials with different disinfectants were subjected to statistical analysis using two sample t-test to find out whether there were any statistically significant difference between two different immersion time intervals (10 minutes and 30 minutes), it was observed that immersion time interval played a very important role in the wettability of impression materials. A higher immersion interval or exposure period caused significant increase in the contact angle values, irrespective of impression materials. Among all disinfectant solutions, for glutaraldehyde and sodium hypochlorite, there were highly significant differences in wettability factor depending on the time of immersion.10 minutes immersion produced lower contact angle values. However the change in the contact angle measurements based on immersion time interval (10 minutes \& 30 minutes) was not significant in cases of other two disinfectants namely iodophor and benzalkonium chloride. It was seen that all impression materials subjected to control (distilled water) also showed increase in contact angles based on immersion period, however this increase here may not 
be clinically significant as the differences were less and all of impression materials had contact angle less than 90 degrees making them hydrophilic. From these results it is imperative that immersion time interval should be carefully selected for usage of these disinfectants.

Since practically for all combination of materials and disinfectants the contact angle values differed significantly between two immersion time intervals, two-way-ANOVA test was carried out for different combinations of impression materials and disinfectants alongwith control (distilled water) for each immersion time interval separately, to evaluate if the performance of different impression materials differ statistically significantly from one another as well as to find the same about different disinfectants. Since the test showed significant differences among impression materials as well as different disinfectants, pair wise comparison for impression materials and disinfectants including control was done using the tukey simultaneous test procedure. From the results of two way ANOVA test, we can see that there were significant differences in the mean contact angles among all impression materials. For the 10 minutes immersion interval, the subgroups subjected to sodium hypochlorite gave the lowest contact angle values. Therefore while using sodium hypochlorite as a disinfectant; the recommended time of immersion is 10 minutes. Disinfectant glutaraldehyde closely followed sodium hypochlorite as far as wettability is concerned and 10 minute immersion produced lower contact angles. So it is suggested to use 10 minutes of immersion in $2 \%$ glutaraldehyde solution for effective disinfection without affecting wettability. For the 30 minutes immersion interval, the subgroups subjected to glutaraldehyde gave lowest contact angle values. Disinfectant sodium hypochlorite closely followed glutaraldehyde in terms of contact angle values when 30 minutes immersion time interval was followed. With benzalkonium chloride and iodophor it was seen that for both 10 minutes and 30 minutes of immersion, the contact angle values were higher compared to glutaraldehyde and sodium hypochlorite. Therefore sodium hypochlorite and glutaraldehyde should be preferred over benzalkonium chloride and iodophor as disinfectant solutions for better wettability.
When impression materials with different disinfectant solutions were evaluated for wettability factor, it was seen that vinylsiloxaether had shown the least contact angle values indicating hydrophilicity, followed by polyether and addition silicone.

Disinfection protocols for impression materials should be routinely followed in dental surgeries to avoid cross infection. But care should be taken so that these procedures are not creating dimensional inaccuracies leading to a faulty cast. Selection of a disinfectant for impressions is an individual choice, but to avoid dimensional changes, time of immersion should be taken care of. Based on this study 10 minutes disinfection with either $0.5 \%$ sodium hypochlorite or $2 \%$ glutaraldehyde produced better results compared to 30 minutes immersion. Therefore 10 minutes disinfection protocol with any of the disinfectant solutions mentioned before is recommended for routine disinfection of elastomeric impression materials.

Silicone impression materials are available in various consistencies. In this study, only some of the low viscosity impression materials were evaluated. It is unreasonable to expect similar results with various other brands of impression materials with various consistencies subjected to different immersion protocols with disinfectant solutions. The objective of this study was only to evaluate the effect of disinfectant solutions on wettability of elastomeric impression materials. The other factors like dimensional changes and effect of different concentrations of various disinfectant solutions on wettability were not investigated. Further research can be taken up to overcome these limitations.

\section{Conclusions}

Within the limitations of this study, the following conclusions can be drawn:

1. All disinfectant solutions decreased the wettability (increased contact angle values) of all the impression materials evaluated irrespective of immersion time interval.

2. The mean contact angle values of addition silicone were highest among elastomers for both 10 minutes and 30 minutes immersion time interval followed by polyether and vinylsiloxaether. 
3. Among all disinfectant solutions, immersion in $2 \%$ glutaraldehyde and $0.5 \%$ sodium hypochlorite revealed highly significant differences $(\mathrm{P}<0.0001)$ in contact angle values compared to $0.05 \%$ iodophor and $0.25 \%$ benzalkonium chloride depending on the duration of immersion. 10 minutes immersion produced lower contact angle values.

4. The mean contact angle values differed significantly depending on the immersion time interval among all impression materials. The results were highly significant $(\mathrm{P}<0.0001)$ for the 10 minutes immersion interval. Disinfection with $0.5 \%$ sodium hypochlorite solution had shown lowest contact angle values followed by $2 \%$ glutaraldehyde, $0.25 \%$ benzalkonium chloride and $0.05 \%$ iodophor respectively.

5. The contact angle values increased considerably when subjected to 30 minutes of immersion with different disinfectants and the results were highly significant $(\mathrm{P}<0.0001)$. Disinfection with $2 \%$ glutaraldehyde had shown lowest contact angle values followed by $0.5 \%$ sodium hypochlorite solution, $0.25 \%$ benzalkonium chloride and $0.05 \%$ iodophor respectively.

6. Among the elastomeric impression materials evaluated, wettability wise vinylsiloxaether proved to be the best impression material followed by polyether and addition silicone.

\section{Source of Funding}

None.

\section{Conflict of Interest}

None.

\section{References}

1. Centers of Disease Control and Prevention (CDC).

Guidelines for infection control in dental health-care settings, 2003. MMWR Recommendations and Reports. 19 December 2003/52 (RR-17), 1-61. Available at: http://www.cdc.gov/ mmwr/preview/mmwrhtml/rr5217a1/htm (accessed on 29 May 2011).

2. Patrick NW. Infection control in fixed prosthodontics. Dent Clin North Am 1992;36:809-31.
3. Craig RG, Powers JM. . Restorative dental materials. 11th edn. St Louis, MO: Mosby; 2002:331-32, 335, 339-40, 366.

4. Anusavice KJ. Phillips' science of dental materials. 11th edn. Philadelphia, PA: Saunders; 2003:224-26.

5. Block SS. Disinfection, Sterilization, and Preservation. Lippincott Williams \& Wilkins. 2001. 5th edition. 3-30, 1049-68.

6. Kotsiomiti E, Tzialla A, Hatjivasiliou K. Accuracy and stability of impression materials subjected to chemical disinfection - a literature review. J Oral Rehabilitation 2008;35:291-99.

7. Davis BA, Powers JM. Effect of immersion disinfection on properties of impression materials. J Prosthodont 1994;3:31-4.

8. Pratten DH, Covey DA, Sheats RD. Effect of disinfectant solutions on the wettability of elastomeric impression materials. J Prosthet Dent 1990;63:223-7.

9. Adabo GL, Zanarotti E, Fonseca RG, Cruz CA. Effect of disinfecting agents on dimensional stability of elastomeric impression materials. J Prosthet Dent 1999;81:621-4.

10. Milward PJ, Waters MG. The effect of disinfection and a wetting agent on the wettability of addition-polymerized silicone impression materials. J Prosthet Dent 2001;86:165-7.

11. Lepe X, Johnson GH, Berg JC, Aw TC, Stroh GS. Wettability, imbibition, and mass change of disinfected low viscosity impression materials. $J$ Prosthet Dent 2002;88:268-76

12. Khalid MA, Edward CC, James SH. The effect of disinfectants on the properties of dental gypsum, Part 2: surface properties. J Prosthodont 2002;11:234-40.

13. Petrie CS, Walker MP, O’Mahony AM, Spencer P. Dimensional accuracy and surface detail reproduction of two hydrophilic vinyl polysiloxane impression materials tested under dry, moist and wet conditions. J Prosthet Dent 2003;90:365-72.

14. Abdelaziz KM, Hassan AM, Hodges JS. Reproducibility of Sterilized Rubber Impressions. Braz Dent J 2004;15(3): 209-13.

15. Khalid MA, Edward CC, James SH. The wetting of surface treated silicone impression materials by mixes containing disinfectants and modifiers. J Prosthodont 2005;14(2):1049.

16. Sheila RSP, Vanderlei LG, Luiz AP, Carla CBS. Analysis of three disinfectants after immersion of irreversible hydrocolloid and ZOE paste impressions. Braz J Oral Sci 2006;5(18);1094-1100.

17. Jagger DC, Vowles RW, McNally L, Davis F, O'Sullivan DJ. The effect of a range of disinfectants on the dimensional accuracy and stability of some impression materials. Eur J Prosthodont Rest Dent 2007;15(1):23-8.

18. Kugel G, Klettke Thomas, Goldberg JA, Benchimol J, Perry RD, Sharma S. Investigation of a New Approach to Measuring Contact Angles for Hydrophilic Impression Materials. J Prosthodont 2007;16:84-92. 
19. Bustos J, Herrera R, González U, Martínez A, Catalán A. Effect of immersion disinfection with $0.5 \%$ sodium hypochlorite and $2 \%$ glutaraldehyde on alginate and silicone: microbiology and SEM Study. Int J Odontostomat 2010;4(2):169-77.

20. Blalock JS, Cooper JR, Rueggeberg FA. The effect of chlorine- based disinfectant on wettability of a vinyl polysiloxane impression material. J Prosthet Dent 2010;104:333-41.
How to cite this article: Kumar VR, Viswambaran M, Dua P, Prakash P. A comparative study on the effect of four disinfectant solutions on wettability of three types of elastomeric impression materials with two different immersion time: An in-vitro study. Ann Prosthodont Restor Dent 2019;5(4):104-13. 